\title{
Null-Controllability and Uniqueness of Optimal Trajectory for Controllable Systems
}

\author{
S.E. Aniaku ${ }^{1}$, P.C. Jackreece ${ }^{2}$ \\ ${ }^{1}$ Department of Mathematics, University of Nigeria, Nsukka, Nigeria. \\ ${ }^{2}$ Department of Mathematics/Statistics, University of Port-Harcourt, Rivers State, Nigeria.
}

Abstract: In this paper, it is shown that in a linear control system which can be steered to zero target from different initial points, the ultimate trajectory is unique provided the control function is bang-bang.

Keywords: Bang-bang control Null-controllability, optimal trajectory,

\section{Introoduction}

Let $E^{n}$ denote the n-dimensional Euclidean space. In $E^{n}$ we consider the linear control system

$$
\dot{x}(\mathrm{t})=A(t) x(t)+B(t) u(t)
$$

$$
x(0)=x_{0}
$$

where $\varepsilon E^{n}, \mathrm{~A}$ and $\mathrm{B}$ are $n \times n$ and $n \times m$ continuous linear matrix functions respectively on $E^{+}=[0, \infty)=\mathrm{I}$, say, and $\mathrm{u}$ is an m-vector valued measurable function with values $u(t)$ lying in a compact, convex set $\Omega$ of $E^{m}$. Such a u is said to be admissible.

A physical system $x$ is said to be controllable if with the aid of external or in-built mechanism (which we call control or input function denoted by $\mathrm{u}$ ) the system can be transferred from state $x_{0}$, say, to another state $x_{1}$ in a finite time $t>0$. If the state $x_{1}$ to which the system is eventually transferred to is zero (that is the origin in the case of the Euclidean space $E^{n}$ ), we say that the system is null-controllable.

Null-controllability is very very important and indeed needed in human situations. For example, the primary aim of many Government and even non-governmental organizations e.g. NAFDAC, WHO, to mention but a few, try to reduce to zero the number of tuberculous patients, the number of HIV/AIDS patients e.t.c in a finite time $t>0$ with the help of subsidized effective drugs for the ailment. With the advances in the means of transportation e.g. cars, aeroplanes, ships motorcycles, the number of the people $x$ dying in mishaps has been increasing in recent years. Various mechanisms are being put to such means of transportation in a view to reducing to zero the number of causalities $x$ in a finite time $t>0$.

Generally, if $x$ is any unwanted object or situation in any community, the desirability and urgency to stamp it out (null-controllability) is very very necessary. For instance, $x$ may denote the number of dangerous criminals in a society, frequent power cut in a certain community or the number of building collapsing in a town. Null- controllability of $x$ in each of these situations is not only necessary but very important. This is why the topic of null-controllability has been very important and has an increasing interest to researchers such as Chukwu [1], Schitendorg [2] and Eke [3] For the system (1.1) above, the subset $C^{m}$ of $E^{n}$ is the mdimensional unit cube, where $C^{m}=\left\{u: u(t) \varepsilon \Omega,\left|u_{j}\right| \leq 1, j=1,2,3 \ldots m\right\}$.

Note that the absolutely continuous solution of (1.1) will be denoted by $x(t, u)$ and is

$$
x(t, u)=X(t) x_{0}+X(t) \int_{0}^{t} X^{-1}(s) B(s) u(s) d s
$$

where $X(t)$ is a fundamental matrix solution of the system (1.1) for $\mathrm{B}=0$ and $X(0)=I$, the identity matrix. condition

The null-controllability, according to Ek e is archived by imposing the condition of (1.1) the boundary

$$
T x=0
$$

Here, it is expected that $T$ is a bounded linear operator defined on $C\left[E^{+}, E^{n}\right]$, the space of all bounded and continuous operators from $E^{+}$to $E^{n}$.

\section{Definition 1}

In the control systems as (1.1) above, if the control function u assumes its maximum value or power, then it is called optimal, and so can be denoted by $u^{*}= \pm 1$. This control $u^{*}$ is called a bang-bang control. 


\section{Definition 2}

The systems (1.1) is said to be Euclidean controllable if for each $x_{0} \in E^{n}$ and $x_{1} \in E^{n}$ each, there exists an admissible control $\mathrm{u}$ and finite time $t_{1}>0$ such that the solution $x(t, u)=x(t)$ of $(1.1)$ satisfies $x(0)=$ $x_{0}$ and $x\left(t_{1}\right)=x_{1}$.

Definition 3 In the definition 2 above, if $x_{1}=\overline{0}$, then we say, that the systems (1.1) is Euclidean nullcontrollable.

We say that an object such as control $u$ or any other concept is optimal if it is adjusted to be the best possible in the concept of prevailing circumstances.

Definition 4 (Trajectory).

The path or locus along which the control function $u(t)$ can steer a point from one point to another in the space $E^{n}$ is called trajectory. This path or locus is usually denoted by G. When this trajectory is a track of optimality achievement, then it is called optimal trajectory.

We now consider the following Theorem which will help us to establish our goal.

Theorem 1 (Lee and Marcus [4])

Consider the autonomous linear process (1.1) in $\mathcal{R}^{n}$, with compact restraint set $\Omega C \mathcal{R}^{m}$,initial state $x_{0}$ and the origin as a the fixed target in $\mathcal{R}^{n}$. Assume

(a) $\mathrm{U}=0$ lies in the interior of $\Omega$,

(b) (1.1) is controllable ,

(c) A is stable, that is each eigenvalue $\lambda$ of A satisfies $\operatorname{Re} \lambda<0$. Then there exists a minimal time optimal controller $u^{*}(t) C \Omega$ on $0 \leq t \leq t^{*}$ steering $x_{0}$ to the origin.

Proof

We know that in (1.1) (i). $\mathrm{u}=0$ lies in the interior of $\Omega$, (ii). (1.1) is controllable and (iii). A is stable ; then the domain of null-controllability is in $\mathcal{R}^{n}$. Then, there exists a controller $\mathrm{u}(\mathrm{t}) c \Omega$ on $0 \leq t \leq t_{1}$ steering $x_{0}$ to the origin. Because the co-domain is a compact target set $\mathrm{G}(\mathrm{t})$ on $0 \leq t \leq t_{1}^{*}$ and controller $\mathrm{u}(\mathrm{t}) C \Omega$ on $0 \leq t \leq t_{1}^{*}$ steering $x_{0}$ to $\mathrm{G}\left(t_{1}\right)$, then we have an optimal controller $u^{*}(t) C \Omega$ on $0 \leq t \leq$ $t^{*}$ steering $x_{0}$ to $G\left(t^{*}\right)$.

In the problem under study, we shall assume that $\mathrm{n}=2$ and $\mathrm{m}=1$ for ease of understanding of the trajectory through which the control function $\mathrm{u}$ transfers the systems (1.1) from a given initial point $x_{0}$, say, to the origin of the $\mathrm{x}-\mathrm{y}$ Cartesian co-ordinate plane. However, not regarding this assumption, the result of this paper can be generalized to arbitrary integral values of $n$ and $m$. Having these conditions in mind, we are now ready to state and prove our main result of this paper.

\section{Main Theorem}

In what follows, we shall assume that the control systems is defined in $E^{2}$ and extreme values of $u^{*}$ is bang-bang control, steering the given systems (1.1) from any suitable initial point $x_{0}$ to zero $\overline{0}$ in finite time $t_{1}>0$. The problem we are interested in solving, is there fore, the following: Theorem.

Consider the control systems (1.1) in $E^{2}$, that is

$$
\begin{gathered}
\dot{x}(t)=A \dot{x}(t)+B u(t) \\
x(0)=x_{0}
\end{gathered}
$$

where $x \in E^{2}$,A and $\mathrm{B}$ are respectively $2 \times 2$ and $2 \times 1$ matrices. If $u^{*}$ is an admissible optimal control which is bang-bang and steers the systems (2.1) from any initial point along a suitable locus to the origin in a finite time $t_{1}>0$, the resulting ultimate optimal trajectory $\Gamma^{*}$ of null-controllability is unique.

Proof:

We know that the systems (2.1) is controllable. Since our $u^{*}= \pm 1$ is bang-bang, then it is normal. Then the convex restraint set $E^{2} C E^{m}$ contains $\mathrm{u}=0$ in its interior, and we have the target $\mathrm{G}$ as the origin $\mathrm{x}=$ 0 . We know that (2.1) satisfies the normality condition since $\mathrm{u}$ is bang-bang. Then for each point $x_{0}$ in the domain of null- controllability $E^{2}$, there exists a unique extremal controller $u^{*}(t)$ steering $x_{0}$ to the origin, and $u^{*}(t)$ is the optimal controller.

Also, if A is stable, then from $E^{2}=E^{n}$, and so each point $x_{0} \in E^{n}$ can be steered to the origin by just one extremal controller $u^{*}(t)$, namely the optimal controller.

Example.

Consider the autonomous control process in $E^{2}$.

$$
\begin{aligned}
& \dot{x}=A x+B u \\
& x(0)=x_{0}
\end{aligned}
$$


In which $x=\left(\begin{array}{l}x_{1} \\ x_{2}\end{array}\right), A=\left(\begin{array}{cc}0 & 1 \\ 0 & -1\end{array}\right), B=\left(\begin{array}{l}1 \\ 1\end{array}\right)$,

Then (2.1) takes the standard form

$$
\begin{aligned}
\left(\begin{array}{l}
\dot{x}_{1} \\
\dot{x}_{2}
\end{array}\right)= & \left(\begin{array}{cc}
0 & 1 \\
0 & -1
\end{array}\right)\left(\begin{array}{l}
x_{1} \\
x_{2}
\end{array}\right)+\left(\begin{array}{l}
1 \\
1
\end{array}\right) u \\
& x(0)=0
\end{aligned}
$$

Note that at extremal point, we have

$$
\begin{aligned}
& x_{2}+u=0 \stackrel{\text { yields }}{\longrightarrow} x_{2}=-u \\
& -x_{2}+u=0 \stackrel{\text { yields }}{\longrightarrow} x_{2}=u
\end{aligned}
$$

with restraint set $\{u \in \Omega:|u| \leq 1\}$ in $E^{1}$. We wish to synthesize the minimal line $x_{1}=0$ with additional requirement that the response can thereafter be held on this line. Thus the target set is

$$
G=\operatorname{Core}\left\{x_{1}=0\right\} .
$$

If the response lies on $x_{1}=0$, then $\dot{x}_{1}=0, x_{2}(t)=-u(t)$ and so $\left|x_{2}\right| \leq 1$.

Conversely, each point $x_{1}=0,\left|x_{2}\right| \leq 1$ can be cntrolled in a set $\left|x_{2}\right| \leq 1$ by

$$
u(t)=-x_{2_{0}} e^{-2 t} \text { for } t \geq 0 \text {. }
$$

Thus $\mathrm{G}$ is the set $\left\{x_{1}=0,\left|x_{2}\right| \leq 1\right\}$. We note that $\mathrm{G}$ is a compact convex set in $E^{2}$ and also that each point $\left[x_{0_{1}}, x_{0_{2}}\right] \in \square$ can be steered to $\mathrm{G}$ by non-extremal controller

$$
u(t)=-x_{2_{0}} e^{-2 t} \text { for } t \geq 0 .
$$

Using the coefficient matrices $A=\left(\begin{array}{cc}0 & 1 \\ 0 & -1\end{array}\right), B=\left(\begin{array}{l}l \\ 1\end{array}\right)$, the vector $\mathrm{w}=1$ along $\Omega$, we see that the normality condition is satisfied. Thus (2.2) is controllable and by theorem 1 we assert that the domain of null-controllability is all $E^{2}$ and that each initial point in $E^{2}$ can be steered to $\mathrm{G}$ by a unique extremal controller $u(t)$. This extremal controller is optimal and the track is known as optimal trajectory.

\section{References}

[1]. E. N. Chukwu, "On the Null-Controllability of Nonlinear Delay Systems with Rstrained control," J. M. Anal, vol. 78, pp. 283-299, 1990.

[2]. W. F. Schmitendorf, "Null Controllability with constraned controls," SIAM J. Control Optim, vol. 18, pp. 327-345, 1980.

[3]. A. N. Ekr, "Null Controllability criteria for Nonlinear systems," NIJOTECH, vol. 7, no. 1, pp. 71-75, 1983.

[4]. E. Lee and L. Markus, Foundations of Contol Theory, New York: John Wiley, 1967.

[5]. J. U. Onwuatu, "Null Controllability of nonlinear infinite Neutral systems," Kyber Netica, vol. 29, no. 4, pp. 325-336, 1993.

[6]. A. N. Eke, "Null Controllability for linear Control systems," Journal Inst Math\& Comp Sc(Math Ser), vol. 3, no. 2, pp. 149-154, 1990.

[7]. R. F. Brammer, "Controllability In linear Autonomous Systems with Positive Contrllers," SIAM J. Control, vol. 10, no. 2, pp. 339$353,1972$.

S.E. Aniaku. "Null-Controllability and Uniqueness of Optimal Trajectory for Controllable Systems." IOSR Journal of Mathematics (IOSR-JM) 13.3 (2017): 49-51. 